\title{
Questionários online: análise comparativa de ferramentas para a criação e aplicação de $e$-surveys
}

\author{
Catarina Neves ${ }^{1}$, Cláudia Augusto ${ }^{2}$, Ana Lúcia Terra ${ }^{3}$ \\ ${ }^{1}$ Instituto Politécnico do Porto (IPP), Porto, Portugal. ORCID: https://orcid.org/0000-0001-8885-549X \\ 2 Instituto Politécnico do Porto (IPP), Porto, Portugal. ORCID: https://orcid.org/0000-0001-9367-6488 \\ ${ }^{3}$ Instituto Politécnico do Porto (IPP), Porto, Portugal. ORCID: https://orcid.org/0000-0003-1292-2849
}

Autor para correspondência/Mail to: Ana Lúcia Terra, anaterra@iscap.ipp.pt

Recebido/Submitted: 12 de agosto de 2020; Aceito/Approved: 22 de setembro de 2020

Copyright (c) 2020 Neves, Augusto \& Terra. Todo o conteúdo da Revista (incluindo-se instruções, política editorial e modelos) está sob uma licença Creative Commons Atribuição-NãoComercial-Compartilhalgual 3.0 Não Adaptada. Ao serem publicados por esta Revista, os artigos são de livre uso em ambientes educacio-

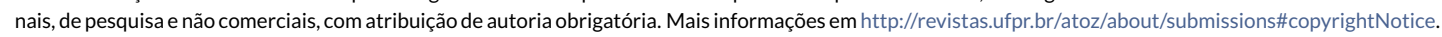

\begin{abstract}
Resumo
Introdução: a disponibilidade de uma grande quantidade de ferramentas para a coleta de dados através de questionários online (e-surveys) oferece novas oportunidades de escolha aos utilizadores, mas também dificulta o processo de seleção. Neste sentido, torna-se relevante mapear as ferramentas existentes e analisá-las à lente de critérios objetivos uniformes. Metodologia: foi realizada uma pesquisa exploratória, com base em dados secundários, para mapeamento das ferramentas de e-surveys existentes. Foi criada e aplicada uma grelha de análise a 15 ferramentas selecionadas num mapeamento inicial. Foi criado um quadro de avaliação comparativa e de pontuação aplicado a dez ferramentas escolhidas dentre as 15 analisadas. Resultados: É proporcionado um levantamento sistemático e atualizado das ferramentas de $e$-surveys existentes e apresentada uma avaliação pontuada das suas principais funcionalidades. São sintetizadas as principais características e funcionalidades das três ferramentas melhor pontuadas (SurveyMonkey, SurveyGizmo e SoGoSurvey), proporcionando aos potenciais utilizadores dados relevantes para que façam uma escolha informada. Conclusão: É evidenciada a necessidade de uma avaliação metódica das ferramentas de e-surveys, dada a existência de muitas ferramentas com características semelhantes. Destaca-se a importância de atender aos condicionalismos do utilizador e aos objetivos da aplicação do questionário. A presente pesquisa terá elevadas potencialidades de uso prático por parte de investigadores, estudantes ou profissionais do setor privado que necessitem de trabalhar com e-surveys.
\end{abstract}

Palavras-chave: Questionários online; Análise comparativa; Ferramentas de coleta de dados.

\begin{abstract}
Introduction: the availability of a wide range of tools for collecting data through online surveys (e-surveys) offers new opportunities for users to choose them. However, it also makes the choice process more difficult. It becomes relevant to map the best existing tools and analyze them through the lens of uniform objective criteria. Method: it carries out an exploratory search, based on secondary data, to map the existing e-survey tools. It creates an analysis grid and applies to 15 tools selected from the initial mapping. After, it applies a grid of comparative evaluation and scoring to 10 tools chosen from the 15 analyzed. Results: it provides a systematic and updated survey of the existing e-survey tools and it presents a scored evaluation of its main features. It summarizes the main characteristics and functionalities of the three best-rated tools (SurveyMonkey, SurveyGizmo and SoGoSurvey), providing potential users with relevant data to make an informed choice. Conclusions: it highlights the need for a methodical evaluation of e-survey tools since there are several tools with very similar characteristics. It also highlights the need to consider the user's requirements and the objectives of the questionnaire during this assessment. This paper will have a high potential for practical use by researchers, students or professionals in the private sector who need to work with e-surveys.
\end{abstract}

Keywords: Online surveys; Comparative analysis; Data collection tools.

\section{INTRODUÇÃO}

$\mathrm{Na}$ atual era digital, a Internet perpassa todas as áreas da atividade humana (Schmidt \& Cohen, 2013), o que se traduz num aumento continuado do número de utilizadores e dispositivos conectados, tanto a nível nacional (PORDATA, 2020) como a nível mundial (International Telecommunication Union, 2020). Tem-se assistido ao crescimento e desenvolvimento de novas tecnologias baseadas na web, desde ferramentas que auxiliam na criação e gestão de conteúdo, até àquelas que potenciam a colaboração de utilizadores. A disponibilidade dos mais variados tipos de ferramentas digitais, tanto proprietárias como open-access, alargando as possibilidades de escolha aos utilizadores, com as vantagens inerentes, também acaba por dificultar o trabalho dos utilizadores, que não sabem como escolher a ferramenta mais adequada às suas necessidades. No caso das ferramentas de criação de questionários online (e-surveys), a profusão da oferta é ilustrada pelo número de resultados obtidos quando se faz uma pesquisa por survey tools e pelo número de ferramentas disponíveis identificadas.

Tal como previsto por alguns autores em meados da década passada, nos últimos quinze anos, os questionários online afirmaram-se como uma técnica de coleta de dados amplamente usada para diversos fins, tanto de índole científica e acadêmica, como no contexto empresarial (Evan \& Mathur, 2005; Lozar Manfreda, Batagelj, \& Vehovar, 2005). Como sintetizaram Daikeler, Bošnjak, \& Lozar Manfreda (2020) o sucesso dos questionários online deve-se a um conjunto de fatores, dos quais se destacam: 1) a diminuição das limitações técnicas graças à melhoria das competências digitais dos inquiridos; 2) melhores níveis de cobertura no acesso à Internet; 3) a 
disponibilidade de um maior número de dispositivos com acesso facilitado à Internet (ligações wi-fi) e amigáveis (por exemplo, ecrãs táteis); 4) diminuição dos custos de acesso à Internet; além de que 5) o contato com as pessoas através de outros meios se tornou mais difícil porque, por exemplo, há um número cada vez menor de agregados familiares com telefone fixo.

Neste cenário, o propósito do presente trabalho é analisar ferramentas para criação e aplicação de questionários online e, por meio dessa análise, determinar quais as três mais adequadas para o efeito, tendo em conta as funcionalidades que oferecem e a sua aplicabilidade tanto ao nível da pesquisa acadêmica, como ao nível da sua utilização por empresas para obtenção de dados relativos à satisfação de clientes e/ou funcionários, por exemplo.

Além da introdução, o texto apresenta uma seção de revisão da literatura dedicada à técnica de coleta de dados através de questionário e aos aspetos distintivos dos e-surveys. É, depois, apresentada com detalhe a metodologia concebida e aplicada para levantamento, análise e avaliação das ferramentas de questionários online. Numa terceira seção, são apresentados e discutidos os resultados da apreciação global das ferramentas de e-surveys, com a caracterização das três consideradas com melhores performances face à avaliação a que foram submetidas. O texto termina com as conclusões, em que é apresentada uma reflexão geral do trabalho desenvolvido.

Considerando o uso generalizado de ferramentas de questionários online, o presente artigo pretende traçar um quadro da oferta existente e fazer uma caracterização, complementada com uma abordagem avaliativa, das opções existentes para o efeito. A relevância da temática encontra aqui uma justificativa, reforçada pelo fato de existir pouca bibliografia em língua portuguesa sobre o assunto e também da necessidade da sua atualização (Walter, 2013).

\section{QUESTIONÁRIOS: UMA REVISÃO BIBLIOGRÁFICA}

O questionário pode ser definido como um instrumento de coleta de dados que inclui um conjunto de perguntas com o intuito de coletar informação de um grupo de respondentes (Moreira, 2004). É um instrumento essencial dentro do conjunto de ferramentas destinadas à coleta de dados, uma vez que permite coletar opiniões, dados demográficos ou feedback de maneira direta, e normalmente a um custo reduzido, além de possibilitar a elaboração de estatísticas (Greenlaw \& BrownWelty, 2009). Conforme explica Parizot (2015, p. 85), esta técnica de coleta de dados sustenta abordagens quantitativas, em que se pretende medir a existência de características mas também as suas relações:

O interesse principal da pesquisa por questionário é o de reunir uma grande quantidade de informações, tanto factuais quanto subjetivas, junto a um número importante de indivíduos - a representatividade dessa amostra autorizando inferir a um conjunto da população de estudo os resultados obtidos junto aos pesquisados. O objetivo de tais pesquisas pode ser o de medir a frequência de características (situações, comportamento, opiniões ou atitudes...) em uma população dada, mas em ciências sociais e humanas ele visa principalmente a analisar as relações entre estas características.

Apesar de a criação de um questionário poder parecer simples, é necessário aplicar tempo e esforço, desenvolvendo uma abordagem global, que implica o enquadramento do questionário no âmbito mais geral do projeto/objetivo em que se enquadra. Como bem sublinharam Sue e Ritter (2007, p. 1), "para aplicar questionários da forma mais eficaz, é importante entender que um questionário é um elemento de um processo que se inicia com a definição de objetivos e termina com a análise de dados e relatórios de resultados".

Os questionários devem ser integrados numa perspectiva de Total Survey Design (TDS) já que o enquadramento no projeto (de investigação acadêmica ou científica, de satisfação de clientes, de sondagem, de avaliação etc.) global é um ponto crítico para o êxito do uso do questionário. Neste sentido, importa pôr em prática uma abordagem holística considerando todas as etapas de desenvolvimento e aplicação do questionário. Havendo propostas diferentes, quanto à sequencialização dos procedimentos de uso dos questionários como ferramenta de coleta de dados (Gil, 2002), consideramos como ajustada a visão de Sue e Ritter (2007). Segundo a abordagem delineada por estas autoras, é necessário atender a oito etapas inerentes ao uso do questionário como técnica de coleta de dados:

a) Definir objetivos: determinar o que se pretende saber e por que, projetar a quem os resultados vão interessar e atender aos custos da coleta de dados;

b) Definir o universo ou população e escolher uma amostra: usar uma amostra já existente ou criá-la, selecionar métodos de amostragem probabilística e não probabilística para criar a amostra;

c) Conceber a estratégia de coleta de dados: avaliar o tempo e os recursos disponíveis, escolher uma modalidade de administração do questionário;

d) Desenvolver o questionário: redigir as perguntas e testar o questionário;

e) Coletar os dados: monitorizar as respostas e usar lembretes, se necessário/aplicável; 
f) Gerir os dados: criar uma codificação, importar ou exportar os dados, limpar os dados, transformar/trabalhar os dados;

g) Analisar os dados;

h) Disseminar os resultados: escrever um relatório ou fazer uma apresentação oral, por exemplo.

As principais características da pesquisa por questionário abrangem a padronização na aplicação e a comparabilidade das respostas (Parizot, 2015). A padronização implica que as respostas similares dadas por pessoas diferentes são consideradas como equivalentes no momento da análise. Por conseguinte, é necessário colocar exatamente as mesmas perguntas ao conjunto de pessoas inquiridas e também homogeneizar o ambiente de aplicação do questionário, porque as condições da sua realização são sempre suscetíveis de influenciar as respostas. As diferenças nas respostas refletem diferenças relativas ao que os inquiridos têm a dizer, e não diferenças nos estímulos resultantes da aplicação do questionário. Nesse sentido, os inquiridos entendem as questões da mesma maneira e produzem respostas com significados similares e, portanto, comparáveis.

Assim, um dos aspetos críticos do uso dos questionários está relacionado com a formulação das perguntas. Redigir um questionário consiste em transformar as questões de pesquisa em indicadores e depois em perguntas que serão colocadas aos inquiridos (Parizot, 2015). Estas questões podem assumir duas formas: questões fechadas (proporcionam ao inquirido a escolha da resposta de entre um conjunto de opções fornecidas pelo pesquisador) e questões abertas (permitem ao inquirido formular a resposta que entender). Quanto ao seu conteúdo, as questões podem incidir sobre "fatos"; sobre crenças, atitudes, opiniões, julgamentos; sobre comportamentos; ou sobre os motivos de certos comportamentos, atitudes (Moreira, 2004).

Além destes aspetos relativos à forma e ao conteúdo dos questionários, numa perspectiva de Total Survey Design é também muito importante considerar o modo de distribuição do questionário.

Tradicionalmente, as modalidades de distribuição dos questionários incluíam o uso do correio postal e do telefone mas, a partir do início do século XXI, a disponibilização dos questionários na Web e por meio do correio eletrônico começou a ganhar terreno e o computador passou a ser um instrumento essencial não só no tratamento dos dados, mas também na sua coleta. De fato, em meados do século XX, o uso do computador nas pesquisas por questionário começou com o CATI (computer-assisted telephone interviewing), em que o entrevistador registrava diretamente no computador as respostas dos entrevistados pelo telefone, e evoluiu para o CAPI (computer-assisted personal interviewing), em que o entrevistador recolhe diretamente as respostas de uma pesquisa "face a face" com o entrevistado (Lozar Manfreda et al., 2005; Parizot, 2015).

Atualmente, distinguem-se quatro modalidades de aplicação dos questionários: por correio postal, por telefone, pessoalmente (face a face) e online. Cada uma destas modalidades apresenta vantagens e desvantagens, cabendo ao responsável pela execução da pesquisa por questionário a escolha da melhor opção, de acordo com os objetivos e os recursos disponíveis. No Quadro 1, são elencadas de modo sintético as principais vantagens e desvantagens de cada modalidade, havendo depois uma análise mais aprofundada dos questionários online.

\begin{tabular}{|c|c|c|}
\hline Modalidade de aplicação & Vantagens & Desvantagens \\
\hline Correio Postal & $\begin{array}{l}\text { Baixo custo } \\
\text { Abrangência geográfica alargada } \\
\text { Aem viés do entrevistador } \\
\text { O anonimato permite perguntas } \\
\text { sensíveis }\end{array}$ & $\begin{array}{l}\text { Mestrado acadêmico; } \\
\text { Dobaixa taxa de resposta } \\
\text { Período de resposta longo } \\
\text { Dificuldade em implementar questões de } \\
\text { contingência } \\
\text { Não saber quem responde à pesquisa }\end{array}$ \\
\hline Telefone & $\begin{array}{l}\text { Viés de cobertura limitado } \\
\text { Rapidez das respostas } \\
\text { Permite perguntas complexas } \\
\text { Amplo alcance geográfico }\end{array}$ & $\begin{array}{l}\text { Pode ser confundido com chamadas } \\
\text { de vendas } \\
\text { Intrusivo } \\
\text { Necessidade de monitorização das } \\
\text { Chamadas sem suporte visual }\end{array}$ \\
\hline $\begin{array}{l}\text { Pessoalmente } \\
\text { (entrevista face a face) }\end{array}$ & $\begin{array}{l}\text { Boas taxas de respostas } \\
\text { Permite perguntas complexas } \\
\text { Tolerância a entrevistas complexas }\end{array}$ & $\begin{array}{l}\text { Alcance geográfico limitado } \\
\text { Demorado } \\
\text { Custoso } \\
\text { Viés do entrevistador dificuldade } \\
\text { em abordar perguntas sensíveis }\end{array}$ \\
\hline
\end{tabular}




\begin{tabular}{|c|c|c|}
\hline Modalidade de aplicação & Vantagens & Desvantagens \\
\hline Online & $\begin{array}{l}\text { Baixo custo } \\
\text { Rápido eficiente } \\
\text { Facilidade de implementação } \\
\text { de questões de contingência } \\
\text { Registo direto de dados } \\
\text { Ampla cobertura geográfica }\end{array}$ & $\begin{array}{l}\text { Viés da cobertura } \\
\text { Dependência de software } \\
\text { Desconhecimento de quem responde } \\
\text { ao questionário }\end{array}$ \\
\hline
\end{tabular}

Quadro 1. Comparação das modalidades de aplicação dos questionários) Fonte: Adaptado de Sue \& Ritter, (2007, p. 07).

Os questionários online têm diversas vantagens para a organização ou pessoa que os desenvolve e aplica. Segundo Vasconcellos e Guedes (2007), o custo de elaboração pode ser reduzido ou nulo, a menos que se opte por uma opção mais dispendiosa; os dados são apresentados imediatamente após o questionário ser respondido; facilidade em usar amostras maiores; os dados semelhantes são facilmente agrupados e podem ser apresentados percentualmente, levando a que as divergências se tornem evidentes, facilitando a análise, reduzindo o erro e o tempo de escrita; entre outros.

Além disso, Ilieva, Baron, e Healey (2001) reforçam que a velocidade de coleta de dados é muito superior e não depende da localização geográfica dos participantes, sendo apropriado para abordagens comparativas internacionais. Do ponto de vista do respondente, também podem ser assinaladas algumas vantagens, como a flexibilidade e conveniência, já que pode completar o questionário quando lhe é conveniente, não necessitando de se deslocar para um local controlado; ausência de um entrevistador, levando a que o respondente se sinta mais à vontade para responder com maior sinceridade; possibilidade de incluir novos estímulos (como sons, cores, imagens, animações etc.) a custo baixo ou nulo, visando chamar a atenção do respondente e obter um maior número de respostas (Hayslett \& Wildemuth, 2004; Kays, Gathercoal, \& Buhrow, 2012).

Contudo, tal como sintetizado pelos autores Evan e Mathur (2005, p. 201-202) e Vasconcellos e Guedes (2007, p. 10-11), os questionários online também apresentam desvantagens:

a) Limitação dos respondentes a quem detêm acesso à internet e competências tecnológicas para uso dos dispositivos, podendo inviabilizar uma amostra representativa da população;

b) Impessoalidade e problemas de privacidade;

c) Dificuldade em incluir incentivos para envio da resposta;

d) Uso de formulários pouco atrativos, devido a ineficiências de formatação e de escassa flexibilidade no layout;

e) Problemas de adesão ao questionário, porque os respondentes podem considerá-lo como invasão de privacidade ou "lixo eletrônico", ignorando e/ou eliminando os pedidos de resposta;

f) Baixo índice de resposta, em relação a outros métodos de aplicação de questionário (correio, fax etc.);

g) Baixa confiabilidade nos dados - os respondentes podem distorcer, intencionalmente ou não, as suas respostas e podem interpretar incorretamente as perguntas, levando a um desvio não propositado do resultado;

h) Dificuldade de acesso a um número suficiente de endereços de e-mail válidos para difusão do questionário.

Para o sucesso dos questionários online, evitando respostas apressadas, irrefletidas ou mesmo o abandono no processo de resposta, Guin, Baker, Mechling, e Ruyle (2012) enunciam algumas recomendações, nomeadamente atender ao design do questionário, combinando adequadamente texto, elementos gráficos, componentes de gamificação e funcionalidades visuais. Adicionalmente, importará limitar a extensão do questionário ao mínimo necessário de perguntas, equilibrar o esforço cognitivo exigido ao respondente, evitar provocar ansiedade emocional ao respondente e não o sobrecarregar com solicitações excessivas para participar em questionários.

Naturalmente, para que um questionário online suporte os objetivos da pesquisa ou do projeto em que está enquadrado, a escolha da ferramenta usada para a criação, distribuição, coleta, armazenamento e, por vezes também, tratamento dos dados, é um ponto crítico. Essa escolha tem de ser fundamentada no conhecimento e na avaliação das ferramentas disponíveis. Na verdade, a escolha da ferramenta de e-survey deve depender fundamentalmente das necessidades e das características da pesquisa (Walter, 2013). Assim, no tópico seguinte iremos explicitar a metodologia seguida para realizar uma análise comparativa de ferramentas de questionários online. 


\section{METODOLOGIA PARA A ANÁLISE COMPARATIVA DE FERRAMENTAS}

A metodologia utilizada para efetuar a abordagem comparativa às ferramentas de questionários online baseouse numa adaptação do quadro de análise previamente criado e testado por Marra e Bogue (2006). Foram definidas quatro categorias, relativas às funcionalidades de desenvolvimento, funcionalidades de implementação e funcionalidades de acesso/representação dos dados, além de outros aspectos importantes a ter em conta. Dentro de cada uma destas categorias, foram incluídas as características mais relevantes, conforme ilustrado na Quadro 2. Assim, nas funcionalidades de desenvolvimento foram considerados os tipos de resposta disponíveis e os tipos de itens passíveis de serem adicionados, a inserção de campos escritos com instruções ou outras indicações úteis, a possibilidade de implementação de lógica instrumental, a capacidade de prever/testar o produto final, bem como as opções de formatação do questionário, nomeadamente o design, a ordem das perguntas, o espaçamento ou as margens, entre outros. As funcionalidades de implementação incluem os aspetos relativos ao lançamento do questionário, como a capacidade de editar o instrumento de coleta de dados depois do seu lançamento, o acesso ao URL e/ou a sua incorporação numa mensagem de e-mail, além da monitorização das respostas, com o acesso à evolução do número de respondentes, o seu rastreamento e técnicas para incrementar a taxa de resposta. As funcionalidades de acesso e representação dos dados dizem respeito aos locais de armazenamento dos dados e à possibilidade de download dos dados em diferentes formatos. A última categoria permitiu a identificação de ferramentas open-source, do preço, de aspectos de interface do utilizador, da segurança do servidor para proteção de dados contra intrusões e contra perda de dados além de outras funcionalidades especiais.

\begin{tabular}{|lc|l|}
\hline $\begin{array}{l}\text { Funcionalidades de } \\
\text { Desenvolvimento }\end{array}$ & $\begin{array}{l}\text { - Seleção do tipo de item a adicionar; } \\
\text { - Inserção de campos escritos: instruçães ou outras mensagens; } \\
\text { - Implementação de lógica instrumental: acesso a determinadas perguntas } \\
\text { dependendo da resposta dada em questões anteriores; } \\
\text { - Habilidade de prever e/ou testar o produto final; } \\
\text { - Formatação/Personalização da ferramenta: design, ordem das perguntas, } \\
\text { espaçamento, margens; }\end{array}$ \\
\hline $\begin{array}{l}\text { Funcionalidades de } \\
\text { Implementação }\end{array}$ & $\begin{array}{l}\text { - Implementação de lógica instrumental: acesso a determinadas; } \\
\text { - Permitir aos utilizadores aceder ao instrumento de coleta de dados (edições } \\
\text { após este passo podem levar a perda de dados); } \\
\text { - Permitir acesso ao URL e sua incorporação em e-mail ou como link num } \\
\text { website; } \\
\text { - Monitorização de respostas: quantas submissões, download de dados in- } \\
\text { ternos para rastreamento de certos respondentes, providenciando aos uti- } \\
\text { lizadores informação útil para melhorar a taxa de resposta e assim facultar } \\
\text { melhores dados de avaliação geral; }\end{array}$ \\
\hline $\begin{array}{l}\text { Funcionalidades de } \\
\text { Acesso/Representação } \\
\text { dos Dados }\end{array}$ & $\begin{array}{l}\text { - Permitir o armazenamento dos dados em cloud; } \\
\text { - Permitir o download dos dados em diferentes formatos; }\end{array}$ \\
\hline $\begin{array}{l}\text { Outros Aspetos Rele- } \\
\text { vantes }\end{array}$ & $\begin{array}{l}\text { - Ferramenta open-source ou proprietária; } \\
\text { - Preço; } \\
\text { - Interface de utilizador; } \\
\text { - Segurança do servidor - para proteção de dados; } \\
\text { - Segurança do servidor - para evitar perdas de dados; } \\
\text { - Disponibilização de funcionalidades especiais. }\end{array}$ \\
\hline
\end{tabular}

Quadro 2. Principais características e funcionalidades das ferramentas de questionários online. Fonte: Adaptado de Marra e Bogue (2006).

Este quadro de análise genérica foi aplicado às ferramentas de questionários online para um primeiro contato com as suas características, tendo servido depois para estruturar os critérios de um quadro avaliativo das ferramentas, conforme explicado em seguida.

$\mathrm{Na}$ etapa de levantamento das ferramentas existentes, foram realizadas pesquisas em motores de busca com termos relevantes e consultados sítios web e revistas especializadas em tecnologia e software. Para dar início à pesquisa de ferramentas de questionários, foi usada a seguinte query: "top 10 survey tools", no Google geral. Os resultados proporcionaram o acesso a artigos de revisão de ferramentas de e-surveys publicados em revistas ou sites de tecnologias e software. Este procedimento permitiu entender quais as ferramentas que, de forma geral, obtinham as melhores críticas, as quais selecionamos para uma análise preliminar.

No final deste levantamento, obtiveram-se 75 ferramentas. A escolha das ferramentas às quais foi aplicado o quadro de análise genérica [Quadro 2] baseou-se em vários fatores: a) integração no topo das classificações em vários sites e revistas sobre tecnologia e software; b) a integração noutros artigos de comparação de ferramentas. Assim, após a coleta de 75 ferramentas distintas, seguiu-se a identificação das 15 que eram mais frequentemente 
mencionadas e melhor classificadas: SurveyMonkey, SurveyGizmo, Qualtrics Research Core, SoGoSurvey, Zoho Survey, Formstack, QuestionPro, Proprofs Survey Maker, Survio, LimeSurvey, HostedSurvey, Survicate, Nicereply, SurveyLegend e TypeForm ${ }^{1}$ [Quadro 3].

\begin{tabular}{|l|l|}
\hline Designação da ferramenta & Sítio Web \\
\hline SurveyMonkey & https://pt.surveymonkey.com/ \\
\hline Surveygizmo & https://www.surveygizmo.com/ \\
\hline Qualtrics Research Core & https://www.qualtrics.com/ \\
\hline SoGoSurvey & https://www.sogosurvey.com/ \\
\hline Zoho Survey & https://www.zoho.com \\
\hline Formstack & https://www.formstack.com/ \\
\hline QuestionPro & https://www.questionpro.com \\
\hline Proprofs Survey Maker & https://www.proprofs.com/survey/ \\
\hline Survio & https://www.survio.com \\
\hline LimeSurvey & https://www.limesurvey.org \\
\hline HostedSurvey & https://hostedsurvey.net/ \\
\hline Survicate & https://survicate.com \\
\hline Nicereply & https://www.nicereply.com/ \\
\hline SurveyLegend & https://www.surveylegend.com/ \\
\hline TypeForm & https://www.typeform.com/ \\
\hline
\end{tabular}

Quadro 3. Listagem das ferramentas de e-surveys objeto de análise geral. Fonte: Elaborado pelos autores (2020).

Destas 15 ferramentas, selecionaram-se dez às quais foi aplicado um quadro de avaliação para ferramentas de questionários online [Quadro 4], no intuito de fazer uma pontuação global destas dez ferramentas e de identificar as três com pontuação mais elevada. Para a seleção das dez ferramentas, foi fator eliminatório a impossibilidade de teste gratuito ou a versão gratuita oferecer funcionalidades muito básicas.

Para uma avaliação comparativa objetiva, com base no quadro de análise genérica e no conhecimento obtido a partir do levantamento das ferramentas existentes, de acordo com a informação disponível nos respetivos sites, foi elaborado o quadro com critérios de avaliação [Quadro 4], aos quais foi aplicada uma escala de quatro níveis, de zero (inexistente ou insuficiente) a três (excelente) [Figura 1].

\begin{tabular}{|c|c|c|c|}
\hline $\mathbf{0}$ & $\mathbf{1}$ & $\mathbf{2}$ & $\mathbf{3}$ \\
\hline $\begin{array}{c}\text { Inexistente ou } \\
\text { Insuficiente }\end{array}$ & Suficiente & Bom & Excelente \\
\hline
\end{tabular}

Figura 1. Escala.

Fonte: Elaborado pelos autores (2020).

Neste quadro de avaliação das ferramentas de questionários online, foram considerados 13 critérios de aferição e a cada um foi aplicada uma escala de avaliação específica de quatro níveis. Estes foram definidos com base nas principais características associadas às ferramentas de criação de questionários, sendo que, para integrarem o quadro de avaliação, cada uma das características teria que ser observada durante a análise exploratória dos softwares e passível de ser comparada entre os mesmos. O primeiro critério diz respeito às possibilidades de seleção dos tipos de resposta (resposta de texto, escolha múltipla, caixas de verificação etc.) e permitiu classificar as ferramentas num largo espectro de possibilidades, desde a existência de apenas cinco opções até 15 ou mais opções para os tipos de resposta passíveis de serem usados. O critério relativo à seleção dos tipos de itens a adicionar inclui a opção básica de apenas texto até à opção completa de texto, imagens, páginas e scripts. No que diz respeito à inserção de campos escritos, foi considerada a impossibilidade de inserção de campos escritos e várias gradações na facilidade da sua implementação bem como na disponibilidade de opções do instrumento de edição. A implementação de lógica instrumental ou lógica de ramificação é avaliada numa escala relativa à sua inexistência, mas também à facilidade de uso, quando existe.

A existência do modo de preview e/ou teste é relevante para observar o comportamento do funcionamento do questionário antes de o aplicar, para corrigir eventuais erros, tendo sido avaliado numa escala que prevê a impossibilidade de teste/preview até à coexistência das duas opções e de outras ferramentas de avaliação do questionário. $\mathrm{O}$ critério relativo às opções de formatação permitiu identificar as ferramentas que não disponibilizam opções de formatação personalizada, as que o permitem através de código e as que apresentam ferramentas de formatação básica ou com muitas opções. As opções de personalização constituíram outro critério

\footnotetext{
${ }^{1}$ Todos os links ativos em agosto de 2020.
} 
bastante relevante na medida em que incide nas possibilidades de apresentação gráfica do questionário as quais podem ser inexistentes, incluir um número variável de modelos ou permitir mesmo a criação de modelos.

O critério sobre o acesso a URL/Link para incorporação em e-mail ou site é muito relevante, porque está relacionado com as possibilidades de divulgação do questionário em plataformas digitais e pode apenas disponibilizar um URL/Link ou facultar a incorporação e conexão do questionário em redes sociais e acesso offline. As opções de monitorização de respostas foram avaliadas considerando apenas a possibilidade de download dos dados até à sua visualização em tempo real, com notificações e novas respostas e filtros. O critério relativo ao formato dos dados prevê a impossibilidade de download dos dados até ao download em três ou mais formatos incluindo CVS ou XML.

Nas formas de representação estruturada dos dados foi prevista apenas a existência de texto até à conjugação de texto, gráficos, relatórios e ferramentas de análise estatística. Foi também considerado como critério de avaliação o fato de a ferramenta ser totalmente gratuita ou com várias opções de planos pagos e de período de teste grátis, tendo sido mais valorizada a gratuidade da ferramenta. O último critério a ser considerado foi de alcance mais geral e incide sobre a interface do utilizador, tendo em conta o seu uso intuitivo e caráter amigável.

\begin{tabular}{|c|c|}
\hline Designação do critério & Escala de avaliação \\
\hline $\begin{array}{l}\text { Seleção dos Tipos de Res- } \\
\text { posta }\end{array}$ & $\begin{array}{l}0-\leq 5 \text { Tipos } \\
1->5 \text { Tipos } \\
2->10 \text { Tipos } \\
3->15 \text { Tipos }\end{array}$ \\
\hline $\begin{array}{l}\text { Seleção dos Tipos de Itens a } \\
\text { Adicionar }\end{array}$ & $\begin{array}{l}0 \text { - Apenas Texto } \\
1 \text { - Texto e Imagens } \\
2 \text { - Texto, Imagens e Páginas } \\
3 \text { - Texto, Imagens, Páginas e Scripts }\end{array}$ \\
\hline Inserção de Campos Escritos & $\begin{array}{l}0 \text { - Sem possibilidade de inserção de campos escritos. } \\
1 \text { - Difícil de implementar e instrumento de edição com opções básicas. } \\
2 \text { - Fácil de implementar e instrumento de edição com opções básicas. } \\
3 \text { - Fácil de implementar e instrumento de edição com muitas opções. }\end{array}$ \\
\hline $\begin{array}{l}\text { Implementação de Lógica } \\
\text { Instrumental ou Lógica de } \\
\text { Ramificação }\end{array}$ & $\begin{array}{l}0 \text { - Não é possível } \\
1 \text { - Possível, mas complexa de implementar. } \\
2 \text { - Possível e fácil de implementar. } \\
3 \text { - Intuitiva de implementar. }\end{array}$ \\
\hline $\begin{array}{l}\text { Existência de Modo Pre- } \\
\text { view/Teste }\end{array}$ & $\begin{array}{l}0 \text { - Sem modo Preview/Teste. } \\
1 \text { - Preview apenas. } \\
2 \text { - Preview e Teste. } \\
3 \text { - Preview, teste e outras ferramentas de avaliação do questionário. }\end{array}$ \\
\hline Opções de Formatação & $\begin{array}{l}0 \text { - Sem possibilidades de formatação. } \\
1 \text { - Formatação através de código. } \\
2 \text { - Ferramenta de formatação própria com opções básicas. } \\
3 \text { - Ferramenta de formatação própria com muitas opções. }\end{array}$ \\
\hline Opções de Personalização & $\begin{array}{l}0 \text { - Sem possibilidades de personalização. } \\
1 \text { - Personalização básica. } \\
2 \text { - Diferentes modelos. }\end{array}$ \\
\hline
\end{tabular}

Quadro 4. Grelha de avaliação das ferramentas de questionários online. Fonte: Elaborado pelos autores (2020).

\section{RESULTADOS E DISCUSSÃO}

A partir da leitura da informação disponibilizada nos sítios web das ferramentas, da visualização de tutoriais de uso das ferramentas, quando existentes, e da criação de questionários de teste, foi possível testar as dez ferramentas de acordo com os parâmetros de avaliação definidos. Esta abordagem facultou um conhecimento substantivo das ferramentas de e-surveys, o que permitiu: a) pontuar cada uma das ferramentas e concluir, com base nessa pontuação, quais as três melhores e b) comparar o desempenho de cada ferramenta em cada um dos critérios [Apêndice 2].

A pontuação das ferramentas de acordo com os critérios e parâmetros de avaliação definidos constitui um exercício de síntese avaliativa que facilitará aos potenciais utilizadores selecionarem a ferramenta mais adequada às suas necessidades, conforme as funcionalidades que forem mais relevantes em cada contexto específico.

Dados os limites de extensão do presente trabalho, iremos focar a nossa análise mais detalhada nas três melhores ferramentas do corpus da pesquisa, a saber o SurveyMonkey, o SurveyGizmo e o SoGoSurvey. Não só foram as 
que obtiveram a melhor pontuação (34, 32 e 33 pontos, respetivamente), como também correspondiam às poucas em que nenhuma das funcionalidades tinha sido avaliada como insuficiente ou inexistente [Apêndice 2].

As três ferramentas escolhidas são baseadas em cloud e incluem planos básicos gratuitos por tempo indefinido, exceto o SurveyGizmo cujo período grátis de utilização é de 14 dias. Todas disponibilizam planos pagos pessoais, empresariais e personalizados, através do contato com as respectivas empresas de comercialização. Estas encontram-se em conformidade com o Regulamento Geral sobre a Proteção de Dados (RGPD), além deste, o SurveyMonkey também segue as diretrizes do Consumer Privacy Act da Califórnia (CCPA). Todas permitem a inclusão de Net Promoter Score, que consiste num método de avaliação da satisfação do utilizador em relação a um produto, serviço ou empresa, pertencendo sempre aos planos pagos; o mesmo se aplica à lógica instrumental, ou lógica de ramificação, que consiste num recurso que altera a próxima pergunta ou página para a qual os respondentes serão direcionados, com base na resposta dada à pergunta atual/selecionada.

O SurveyMonkey que apresenta um banco de perguntas, com diversos exemplos; faculta um leque de 20 tipos de perguntas. Quanto à inserção de campos, permite textos, imagens, questões, ações e, até mesmo custom scripts, assim como o SoGoSurvey. Esta ferramenta permite testar e pré-visualizar o questionário, estimando o tempo de conclusão e a taxa de conclusão do mesmo. Em termos de personalização, permite a mudança de cores, a inserção de logotipo, layout, rodapé, pano de fundo e mudança da fonte no texto. O compartilhamento do questionário é possível por Link/URL, pelas redes sociais, incorporação em website e e-mail e comprar respostas segmentadas. A ferramenta também possibilita a compra de respostas de um determinado perfil demográfico, constituindo a única que apresenta este recurso. No que toca à análise de resultados, possibilita criação de regras para a representação da informação e permite fazer o download dos dados nos formatos CSV, PDF, XLS, PPTX e SPSS. Permite também o compartilhamento dos dados através da criação, pela própria ferramenta, de uma URL de compartilhamento, que pode ser distribuída pelo utilizador.

O SurveyGizmo, além dos recursos já referidos, realça-se pelo seu vasto leque de tipos de perguntas e pelo fato de permitir a importação de questionários elaborados em MS Word. As opções de formatação são bastante básicas, consistindo num editor de texto simples e inserção de imagem, áudio e vídeo. Para personalizar, dispõe de opções para seleção de fundos, temas, inserção de logotipos e customização em HTML/CSS. Em termos de compartilhamento do questionário, é possível compartilhar por Link/URL, código QR, pelas redes sociais, incorporação em website e e-mail. Possibilita a criação de relatórios personalizados e download dos mesmos no formato CSV, permitindo a escolha de delimitadores.

Por fim, o SoGoSurvey apresenta mais de 20 tipos de questões. As opções de formatação que oferece são diversas, como mudar o tamanho, forma e cor do texto, inserir hiperligações, inserir marcas, entre outros. A personalização é relativamente escassa, contando com cerca de cinco temas, com paletas de cores predefinidas e básicas; contudo, possibilita a inserção de logotipo e personalização de cabeçalho e rodapé. Além disso, permite retirar uma cópia em PDF ou MS Word, gratuitamente, ou Scanner-Ready, do próprio modelo do questionário por preencher. Em termos de compartilhamento do questionário, é possível compartilhar por Link/URL, código QR, pelas redes sociais, incorporação em website e e-mail. A ferramenta permite a importação de dados com as extensões Excel e CSV. Para análise de dados, é possível a exportação para Excel, CSV, XML, SPSS, Access, MS Word, HTML e SoGoSurvey1, porém, ao contrário das outras duas, os relatórios são criados automaticamente.

É possível, nas três ferramentas, personalizar e visualizar o questionário em três tipos de dispositivos: desktop, tablet e telemóvel, para que o referido não perca a sua forma. Quanto à visualização de resultados, as três permitem a sua monitorização em tempo real, contudo, a aplicação de filtros é apenas possível no SurveyMonkey e no SurveyGizmo.

Como também se observa no quadro, poucas diferenças existem entre estas ferramentas na maior parte dos critérios de avaliação. Como principais pontos de divergência e que podem levar a optar preferencialmente por uma das ferramentas, apontam-se a interface de utilizador, a implementação da lógica instrumental, o modo preview/teste e as opções de monitorização de resposta. Destaca-se que, em termos gerais, o SurveyMonkey é a ferramenta mais completa e equilibrada, cuja pontuação nunca foi menor que Bom em qualquer um dos aspectos, enquanto as outras acabam por abdicar de complexidade e qualidade em algumas funcionalidades em prol de novas ou melhores funcionalidades. Por exemplo, o SurveyGizmo tem uma implementação de lógica instrumental menos intuitiva, mas permite mais formas de compartilhamento do questionário, e o SoGoSurvey tem uma interface de utilizador muito intuitiva, mas mais simples, tendo menos opções de resposta.

\section{CONCLUSÃO}

Em primeiro lugar, este estudo evidenciou a necessidade de definir e aplicar critérios de avaliação e de escolha de uma ferramenta de criação e aplicação de questionários online, considerando o vasto leque de escolhas oferecido pelo mercado e a semelhança das funcionalidades disponibilizadas pelas diferentes ferramentas. Através da aplicação do quadro de avaliação, foi possível a identificação dos pontos fortes e fracos de cada uma, ajudando à decisão do utilizador, já que se pontuaram as diferentes funcionalidades das ferramentas, o que as destacou umas das outras, numa perspectiva comparativa. 
Verificou-se também que as melhores ferramentas apenas dão acesso a todas as suas funcionalidades com a adesão a um plano pago, pelo que apenas se justifica o seu uso em situações em que determinadas funcionalidades são imprescindíveis (por exemplo, a implementação de lógica instrumental), sendo que, para fins mais simples, o utilizador pode optar por uma solução financeiramente mais amigável.

Assim sendo, mesmo tendo identificado as três melhores ferramentas, destaca-se a importância que as necessidades do utilizador e objetivo do questionário terão na tomada de decisão em relação a qual delas usar. Além disto, é preciso ter em conta que, mesmo reconhecendo a utilidade e potencial deste tipo de software, é essencial um bom planeamento e delimitação de objetivos para a qualidade do trabalho que se quer realizar, uma vez que, apenas por si, o uso destas ferramentas não garante alcançar os resultados pretendidos no projeto ou investigação.

Por fim, convém realçar que, apesar das possibilidades oferecidas por estas ferramentas e das grandes vantagens financeiras e temporais da aplicação dos questionários online, em alguns casos, mantém-se a relevância do uso combinado das duas modalidades (online e correio postal), de forma não só obter um maior número de respostas como garantir que são abrangidos o maior número possível de grupos demográficos, evitando o enviesamento dos dados.

\section{APÊNDICE A}

\begin{tabular}{|c|c|c|c|c|c|c|c|c|c|c|c|c|c|c|}
\hline \multirow{2}{*}{\multicolumn{2}{|c|}{$\begin{array}{l}\text { Software / Caracteristicas e } \\
\text { Funcionalidades }\end{array}$}} & \multicolumn{7}{|c|}{ Funcionalidades de Desenvolvimento } & \multicolumn{2}{|c|}{$\begin{array}{l}\text { Funcionalidades de } \\
\text { Implementação }\end{array}$} & \multicolumn{2}{|c|}{$\begin{array}{c}\text { Funcionalidades de } \\
\text { Acesso/Representação } \\
\text { dos Dados }\end{array}$} & \multicolumn{2}{|c|}{ Outros Aspetos } \\
\hline & & \multirow{2}{*}{$\begin{array}{c}\begin{array}{c}\text { Seleçăo dos } \\
\text { Tipos de } \\
\text { Resposta }\end{array} \\
3 \\
3\end{array}$} & \multirow{2}{*}{$\begin{array}{c}\text { Seleçăo de } \\
\text { Tipos de Itens a } \\
\text { Adicionar }\end{array}$} & \multirow{2}{*}{\begin{tabular}{|c|}
$\begin{array}{c}\text { Inserçăo de } \\
\text { Campos Escritos }\end{array}$ \\
3 \\
\end{tabular}} & \multirow{2}{*}{\begin{tabular}{|c}
$\begin{array}{c}\text { Implementaçăo } \\
\text { de Lógica } \\
\text { Instrumental }\end{array}$ \\
2
\end{tabular}} & \multirow{2}{*}{$\begin{array}{c}\begin{array}{c}\text { Existência de } \\
\text { modo } \\
\text { Preview/Teste }\end{array} \\
3\end{array}$} & \multirow{2}{*}{$\begin{array}{c}\begin{array}{c}\text { Opçöes de } \\
\text { Formataçăo }\end{array} \\
3\end{array}$} & \multirow{2}{*}{$\begin{array}{c}\begin{array}{c}\text { Opçōes de } \\
\text { Personalizaçăo }\end{array} \\
3\end{array}$} & \multirow{2}{*}{$\begin{array}{c}\text { Acesso ao } \\
\text { URL/Link para } \\
\text { incorporaçăo } \\
\text { em e-mail ou } \\
\text { site } \\
\\
2\end{array}$} & \multirow{2}{*}{\begin{tabular}{|c}
$\begin{array}{c}\text { Opçöes de } \\
\text { Monotorizaçăo } \\
\text { de Respostas }\end{array}$ \\
3 \\
3
\end{tabular}} & \multirow{2}{*}{\begin{tabular}{|c|}
$\begin{array}{c}\text { Formatos de } \\
\text { Download dos } \\
\text { Dados }\end{array}$ \\
3 \\
\end{tabular}} & \multirow{2}{*}{\begin{tabular}{|c|}
$\begin{array}{c}\text { Formas de } \\
\text { Representação } \\
\text { Estrutrada dos } \\
\text { Dados }\end{array}$ \\
3 \\
\end{tabular}} & \multirow{2}{*}{$\begin{array}{c}\text { Open-Source ou } \\
\text { Paga } \\
2 \\
\end{array}$} & \multirow{2}{*}{$\begin{array}{c}\text { Interface do } \\
\text { Utilizador }\end{array}$} \\
\hline (a) & SurveyMonkey & & & & & & & & & & & & & \\
\hline srvegizmo & SurveyGizmo & 3 & 3 & 2 & 1 & 3 & 3 & 2 & 3 & 3 & 3 & 2 & 2 & 1 \\
\hline $\mathrm{ITN}_{\mathrm{N}}$ & SoGosurvey & 2 & 3 & 2 & 3 & 1 & 3 & 3 & 2 & 1 & 3 & 2 & 2 & 3 \\
\hline Mena Survey & Zoho Survey & 3 & 2 & 2 & 1 & 2 & 3 & 3 & 3 & 1 & 1 & 2 & 2 & 0 \\
\hline D formititack & Formstack & 2 & 2 & 1 & 0 & 2 & 3 & 3 & 0 & 1 & 3 & 0 & 0 & 1 \\
\hline Equestonfio & QuestionPro & 2 & 1 & 2 & 1 & 2 & 3 & 2 & 0 & 1 & 3 & 1 & 1 & 3 \\
\hline 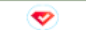 & SurveyLegend & 2 & 2 & 3 & 0 & 1 & 3 & 1 & 2 & 2 & 3 & 1 & 1 & 3 \\
\hline ¿survio & Survio & 2 & 2 & 2 & 2 & 3 & 0 & 2 & 2 & 2 & 3 & 1 & 1 & 3 \\
\hline (2) & LimeSurvey & 3 & 1 & 2 & 0 & 1 & 3 & 1 & 3 & 2 & 3 & 0 & 3 & 1 \\
\hline P vemeart & Survicate & 1 & 0 & 1 & 3 & 1 & 0 & 2 & 1 & 2 & 1 & 1 & 1 & 3 \\
\hline
\end{tabular}

Figura 2. Apêndice A.

Fonte: Elaborado pelos autores (2020). 


\section{REFERÊNCIAS}

Evan, J. R., \& Mathur, A. (2005). The value of online surveys. Internet Research, 15(2), 195-219. doi: 10.1108/10662240510590360.

Gil, A. C. (2002). Como elaborar projetos de pesquisa. São Paulo: Atlas.

Greenlaw, C., \& BrownWelty, S. (2009). A comparison of web-based and paper-based survey methods: testing assumptions of survey mode and response rate. Evaluation Review, 33(5), 464-480. doi: 10.1177/0193841X09340214.

Guin, T. D., Baker, R., Mechling, J., \& Ruyle, E. (2012). Myths and realities of respondent engagement in online surveys. International Journal of Market Research, 54(5), 613-633. doi: 10.2501/IJMR-54-5-613-633.

Hayslett, M. M., \& Wildemuth, B. M. (2004). Pixels or pencils? the relative effectiveness of web-based versus paper surveys. Library and Information Science Research, 26(1), 73-93. doi: 10.1016/j.lisr.2003.11.005.

Ilieva, J., Baron, S., \& Healey, N. M. (2001). Online surveys in international marketing research: pros and cons. In Manchester metropolitan university business school working paper series. Recuperado de https://e-space.mmu.ac.uk/ 1640/1/ilievawp01_10.pdf.

International Telecommunication Union. (2020). The itu ict sdg indicators. Recuperado de https://www.itu.int/en/ ITU-D/Statistics/Pages/SDGs-ITU-ICT-indicators.aspx

Kays, K., Gathercoal, K., \& Buhrow, W. (2012). Does survey format influence self-disclosure on sensitive question items? Computers in Human Behavior, 28(1), 251-256. doi: 10.1016/j.chb.2011.09.007.

Lozar Manfreda, K., Batagelj, Z., \& Vehovar, V. (2005). Design of web survey questionnaires: Three basic experiments. Internet Research, 15(2), 195-219. doi: 10.1108/10662240510590360.

Marra, R. M., \& Bogue, B. (2006). A critical assessment of online survey tools. In Proceedings of the 2006 women in engineering programs and advocates network conference.

Moreira, J. M. (2004). Questionários: teoria e prática. Coimbra: Almedina.

Parizot, I. (2015). A pesquisa por questionário. In $A$ pesquisa sociológica. (p. 85-101). Petrópolis: Vozes.

PORDATA. (2020). Ciência, tecnologia e sociedade de informação: Computadores e internet. Recuperado em 2020, agosto 6, de https://www.pordata.pt/Subtema/Municipios/ Computadores $+\mathrm{e}+$ Internet-227.

Schmidt, E., \& Cohen, J. (2013). A nova era digital: reformulando o futuro das pessoas, das nações e da economia. Lisboa: Dom Quixote.

Sue, V. M., \& Ritter, L. A. (2007). Conducting online surveys. Thousand Oaks: Sage.

Vasconcellos, L., \& Guedes, L. (2007). E-surveys: vantagens e limitações dos questionários eletrônicos via internet no contexto da pesquisa cientáfica. In $X$ semead: Seminários em administração da fea-usp. Recuperado de http://sistema.semead.com.br/10semead/sistema/ resultado/trabalhosPDF/420.pdf.

Walter, O. M. F. C. (2013). Análise de ferramentas gratuitas para condução de survey online. Produção \& Produção, 14(2), 44-58. doi: 10.22456/1983-8026.22172.
Como citar este artigo (APA):

Neves, C., Augusto, C., \& Terra, A. L. (2020). Questionários online: análise comparativa de ferramentas para a criação e aplicação de e-surveys Online surveys: comparative tool analysis for the creation and administration of e-surveys. AtoZ: novas práticas em informação e conhecimento, 9(2), 69 - 78. Recuperado de: http://dx.doi.org/10.5380/atoz.v9i2.75826 